\title{
Effects of Naloxegol on Gastrointestinal Transit and Colonic Fecal Volume in Healthy Participants Receiving Oxycodone
}

\begin{abstract}
Anne E Olesen, ${ }^{1,2,3 *}$ Debbie Grønlund, ${ }^{1,2}$ Esben B Mark, $^{1,2}$ Klaus Krogh $^{4}$ Jens B Frøkjær, ${ }^{2,5}$ and Asbjørn M Drewes ${ }^{1,2}$
Mech-Sense, Departments of ${ }^{1}$ Gastroenterology and Hepatology, and ${ }^{5}$ Radiology, Aalborg University Hospital, Aalborg; Denmark; ${ }^{2}$ Department of Clinical Medicine, Aalborg University, Aalborg, Denmark; ${ }^{3}$ Department of Clinical Pharmacology, Aalborg University Hospital, Aalborg; Denmark; and ${ }^{4}$ Neurogastroenterology Unit, Department of Hepatology and Gastroenterology, Aarhus University Hospital, Aarhus, Denmark
\end{abstract}

\section{Background/Aims}

Opioids cause gastrointestinal (GI) dysmotility, decrease gut secretion, and affect gut sphincters. Symptoms of opioid-induced bowel dysfunction may be alleviated by peripherally acting opioid antagonists like naloxegol, but detailed knowledge on $\mathrm{Gl}$ effects of this drug is lacking. We hypothesized that naloxegol, compared to placebo, would reduce GI transit time and colonic fecal volume in opioid-treated healthy participants.

\section{Methods}

We conducted a randomized, double-blinded, single-center, 2-way cross-over study in 24 healthy males, randomized to a 6 day treatment period of oxycodone (15 mg twice a day) co-administered with either naloxegol (25 mg once a day) or matching placebo. Participants swallowed an electromagnetic capsule which determined Gl transit times. Colonic fecal volume was quantified with magnetic resonance imaging both pre-treatment and post-treatment.

\section{Results}

Naloxegol reduced total GI transit time by $21 \%$ (56 hours vs 71 hours, $P=0.02$ ) and colonic transit time by $23 \%$ (45 hours vs 59 hours, $P<0.01$ ), compared to placebo. However, no difference in colonic fecal volume was found ( $818 \mathrm{~mL} v 884 \mathrm{~mL}, P=0.20)$.

\section{Conclusions}

Short-term administration of naloxegol in healthy participants reverses the retardation of total Gl and colonic transit induced by oxycodone. This supports the use of naloxegol in the treatment of Gl side effects to opioid treatment, and add knowledge to the current understanding of mechanisms behind peripherally-acting opioid antagonists.

(J Neurogastroenterol Motil 2019;25:602-610)

\section{Key Words}

Analgesics, opioid; Constipation; Motility; Naloxegol; Oxycodone

Received: May 9, 2018 Revised: March 22, 2019 Accepted: August 7, 2019

(c) This is an Open Access article distributed under the terms of the Creative Commons Attribution Non-Commercial License (http://creativecommons. org/licenses/by-nc/4.0) which permits unrestricted non-commercial use, distribution, and reproduction in any medium, provided the original work is properly cited.

*Correspondence: Anne E Olesen, MSc (Pharm), PhD

Department of Clinical Pharmacology, Aalborg University Hospital, Mølleparkvej 8a, DK-9000 Aalborg, Denmark Tel: +45-9766-0535, Fax: +45-9766-3577, E-mail: aneso@rn.dk 


\section{Introduction}

Opioids are commonly prescribed to treat pain of moderate to severe intensity. However, opioid treatment may induce a wide range of severe and bothersome gastrointestinal (GI) side effects, collectively termed opioid-induced bowel dysfunction (OIBD). The pathophysiology behind OIBD rely on opioid binding of peripheral $\mu$-opioid receptors in the enteric nervous system. This leads to inhibition of neurotransmitter release in the enteric neurons, which diminishes local propulsive reflexes, increases sphincter tone, and ultimately causes dysmotility. Additionally, stasis of luminal content, and the fact that opioids inhibit vasoactive intestinal peptide release, induce excessive passive absorption of fluids. ${ }^{1}$ Clinically this leads to dry, hard stools accumulating in the colon, causing prolonged transit time. ${ }^{2}$ Thus, the most common and burdensome part of the OIBD complex is constipation, which is reported by $40-70 \%$ of all long-term opioid users. ${ }^{3}$

OIBD significantly deteriorates patients' quality of life, and imposes substantial cost to society. ${ }^{4-6}$ Recommended treatment strategies to alleviate these GI side effects of opioids rely on dietary changes, stool softeners and laxatives. ${ }^{7}$ However, these treatment options do not target the underlying pathophysiology of OIBD. Hence, the use of peripherally acting $\mu$-opioid receptor antagonists, eg, naloxegol, has become increasingly recognized for inclusion in OIBD treatment algorithms. ${ }^{8}$ Naloxegol, a polymer conjugate of the opioid antagonist naloxone, antagonizes opioid receptors in the GI tract, while preserving centrally mediated analgesia. ${ }^{9}$ Until now, most studies have focused on the safety and tolerability of naloxegol, ${ }^{10,11}$ as well as efficacy in terms of reduction in weekly spontaneous bowel movement frequency in opioid-treated patients and healthy participants. ${ }^{12-14}$ However, there is a need to elucidate the more detailed underlying mechanisms of naloxegol, to add information on how this type of drug works to relieve symptoms of OIBD. Employing an experimental OIBD model, we have previously shown that a 5-day treatment of oxycodone prolong GI transit time, increase colonic volume, and cause subjectively assessed symptoms of OIBD. ${ }^{2,15}$ The present study is a continuation of a previously published paper where we demonstrated that naloxegol improved the recto-anal inhibitory reflex and reduced GI symptoms in an experimental model of OIBD. ${ }^{16}$ In the present study, we hypothesized that naloxegol, compared to placebo, would reduce GI transit time and fecal volume in the colon of healthy participants receiving oxycodone. The corresponding aims are to assess total GI transit time, gastric emptying, small bowel transit time and colonic transit time as primary endpoints; segmental colonic transit times, total and segmental colonic fecal volumes as secondary endpoints, during both treatments.

\section{Materials and Methods}

This study is part of a larger protocol that also evaluated the effect of naloxegol on anal sphincter function and GI symptoms. Thus, some of the presented methods have been published previously. ${ }^{16}$ The full protocol is registered in ClinicalTrials. gov (EudraCT No. 2015-000419-42). The Danish Medicines Agency (Reference No. 2015021429) and The North Denmark Region Committee on Health Research Ethics (Reference No. N-20150014) approved the protocol, and the study was carried out in compliance with the European Community rules of Good Clinical Practice, and the International Conference of Harmonization.

\section{Experimental Design}

This randomized, double-blinded, cross-over study was conducted from August 2015 to May 2016 at the Mech-Sense research laboratories, Department of Gastroenterology and Hepatology, Aalborg University Hospital, Denmark. The sample size calculation was based on the variance of total GI transit time as reported in a previous study ${ }^{17}$ where a 12 -hour difference in transit time was found (SD, 9.6 hours). Including this, with a power of $95 \%$ and and $\alpha$ at 0.05 in a 2-sided test, a sample size of 19 participants were found appropriate. To allow for missing data, a sample size of 24 participants was chosen. Randomization was achieved using a computer-generated block-randomization list, and mirrorrandomization was used for replacement of dropouts. Eligible participants were opioid naïve healthy men of Northern European descent, aged 20 years to 60 years. Key exclusion criteria were use of prescription medication, history of substance- or alcohol abuse, and smoking. Participants were invited to a screening visit within 14 days of start of the first treatment period, in which they gave written informed consent.

The study consisted of two 6 day treatment periods, and a $\geq 14$ day washout period was applied (Fig. 1). Treatment periods began with baseline (pre-treatment) measurements of colonic fecal volume using magnetic resonance imaging (MRI), and GI symptoms using self-assessed questionnaires. Hereafter the first dose of oxycodone was administered. Participants received envelopes for homeadministration of either: prolonged-release oxycodone tablets (10 $\mathrm{mg}$ twice a day [bid] on day 1, $15 \mathrm{mg}$ bid on days 2-5, and $15 \mathrm{mg}$ once a day [qd] on day 6) and naloxegol tablets (25 mg qd on days 


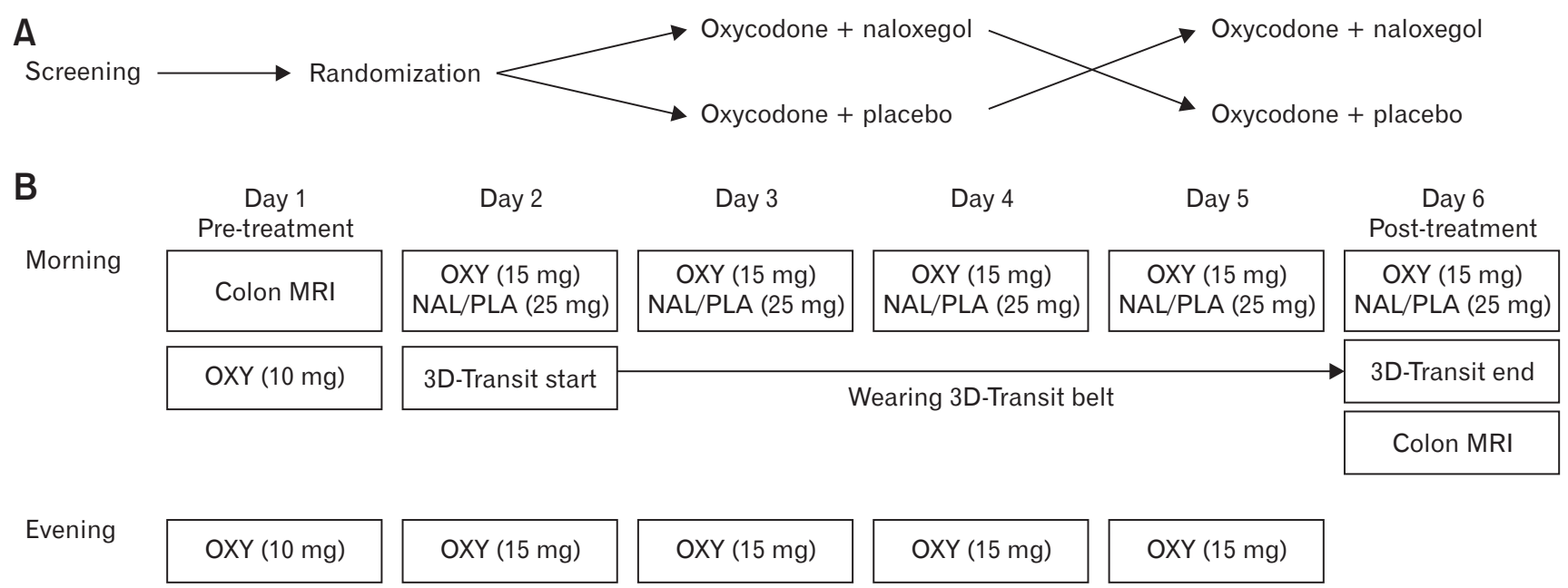

Figure 1. Study design (A) and timeline (B) for a treatment period. MRI, magnetic resonance imaging; OXY, oxycodone; NAL, naloxegol; PLA, placebo.

2-6) (oxycodone + naloxegol), or prolonged-release oxycodone and matching placebo tablets (oxycodone + placebo). Medication was delivered by The Hospital Pharmacy at Aalborg University Hospital, Denmark. On day 2, the 3D-Transit system belt was mounted at home, and the 3D-Transit electromagnetic capsule was swallowed. Throughout days 2-5, participants wore the 3D-Transit belt and self-administrated the medication on scheduled time points. The treatment periods terminated at day 6 (post-treatment), in which the belt was detached at the hospital and assessment of colonic fecal volume was repeated.

\section{Gastrointestinal Transit Time}

Technical specifications of the 3D-Transit system are described in detail elsewhere. ${ }^{18}$ In the morning of day 2 , participants mounted the abdominal belt containing the recording detector plate, and after ingestion of a standardized meal (nutrition bar; $375 \mathrm{kcal}, 11.4$ $\mathrm{g}$ fat, and $1.8 \mathrm{~g}$ fiber) the 3D-Transit electromagnetic capsule was swallowed with a glass of water. Participants were instructed to wear the abdominal belt throughout the rest of the treatment period, only interrupted when showering. They were able to perform their normal daily activities, however, hard physical work was not allowed. The recordings stopped when participants arrived at the research department in the morning of day 6. Here, retention or expulsion of the capsule was confirmed by monitoring emitting signals from the capsule in real-time using 3D-Transit analysis software (Motilis Medica SA, Lausanne, Switzerland). Gastric emptying, small bowel transit time, and colonic transit time were determined manually using the analysis software, by evaluating changes in bowel contraction frequencies observed on capsule rotation graphs, timefrequency map of contractions, and 2D images of capsule position, as described previously. ${ }^{18}$ Total GI transit time was defined as the time between ingestion and expulsion of the capsule. If the capsule was retained, the time of the last confirmed capsule signal was used as the earliest possible expulsion time. Transit times of the 4 colonic segments (cecum/ascending colon, transverse colon, descending colon, and rectosigmoid colon) were assessed based on algorithms of capsule trajectory incorporated into a custom MATLAB (R2015b version 8.6; MathWorks, Inc, Natick, MA, USA) application, as earlier validated. ${ }^{15}$

\section{Colonic Fecal Volume}

Participants were always scanned in a fasting state in the morning. No restrictions on bowel movements were made prior to scanning, and no bowel preparations were used. 3D-Transit capsules are not approved for MRI, which is why imaging was only performed on day 6 in participants in which capsule retention was ruled out. MRI scans were acquired using a GE 3T scanner (GE Signa HDxt, General Electrical, Milwaukee, Wisconsin, USA). A coronal image series of the abdomen was obtained using Dixontype liver accelerated volume acquisition (LAVA-Flex) water-only scans (echo time $[\mathrm{TE}]=2.3 / 4.6$, repetition time $[\mathrm{TR}]=7.2 \mathrm{mil}$ liseconds, in-plane resolution $0.9375 \times 0.9375 \mathrm{~mm}$, slice thickness $4 \mathrm{~mm}$ ). The entire series were performed during a single inspired breath hold of approximately 20 seconds. In total, 35-40 contiguous slices were obtained covering the entire colon and rectum. A semiautomatic in-house data analysis software was used to determine the 
volume of non-gaseous colonic content (feces) as described in detail elsewhere, ${ }^{19,20}$ and as suggested by another research group using a similar approach. ${ }^{21}$ In short, regions of interest were manually outlined on each image encapsulating the following colonic segments; ascending colon (including cecum), transverse colon, descending colon, and rectosigmoid colon. A classification approach using $\mathrm{k}$ means clustering was applied to refine the segmentation of the colon, and calculate the fecal volume.

\section{Gastrointestinal Symptoms}

Results from the Patient Assessment of Constipation Symptom (PAC-SYM) questionnaire and the Bristol Stool Form Scale have been published previously ${ }^{16}$ and were used for analysis of association between subjective and objective measures.

\section{Statistical Methods}

Data were assessed for normality by graphical and numerical methods (histograms, Q-Q plots, and Shapiro-Wilk tests) and handled accordingly. Total/segmental transit times and data on capsule retention were analyzed by Wilcoxon signed rank sum tests (Bonferroni-adjusted for multiple comparisons). Segmental colonic fecal volumes were analyzed by repeated measures mixed models.
Bonferroni-adjusted post hoc tests were applied for all mixed models in which an overall significant difference was found. Pearson's correlation coefficient was used to identify associations between GI symptoms (reported previously ${ }^{16}$ ) and (1) effects on GI transit time and (2) effects on colonic fecal volumes. All reported $P$-values were 2-tailed and values $<0.05$ were considered statistically significant. Data were analyzed using Stata version 14.0 (StataCorp LP, Texas, TX, USA). Descriptive results are presented as means $\pm \mathrm{SD}$.

\section{Results}

One participant was excluded after the first treatment period (due to non-adherence to the study protocol). He was replaced, and thus, 24 participants completed both treatment periods (Fig. 2). Age ranged from 20-46 years, and body mass index ranged from $20.9-31.5 \mathrm{~kg} / \mathrm{m}^{2}$.

\section{Gastrointestinal Transit Time}

Total and segmental GI transit times are presented in Table 1. Recordings of gastric emptying, small bowel transit time, colonic transit time, and total GI transit time were obtained in all participants in both treatment periods (48 recordings in total). However,

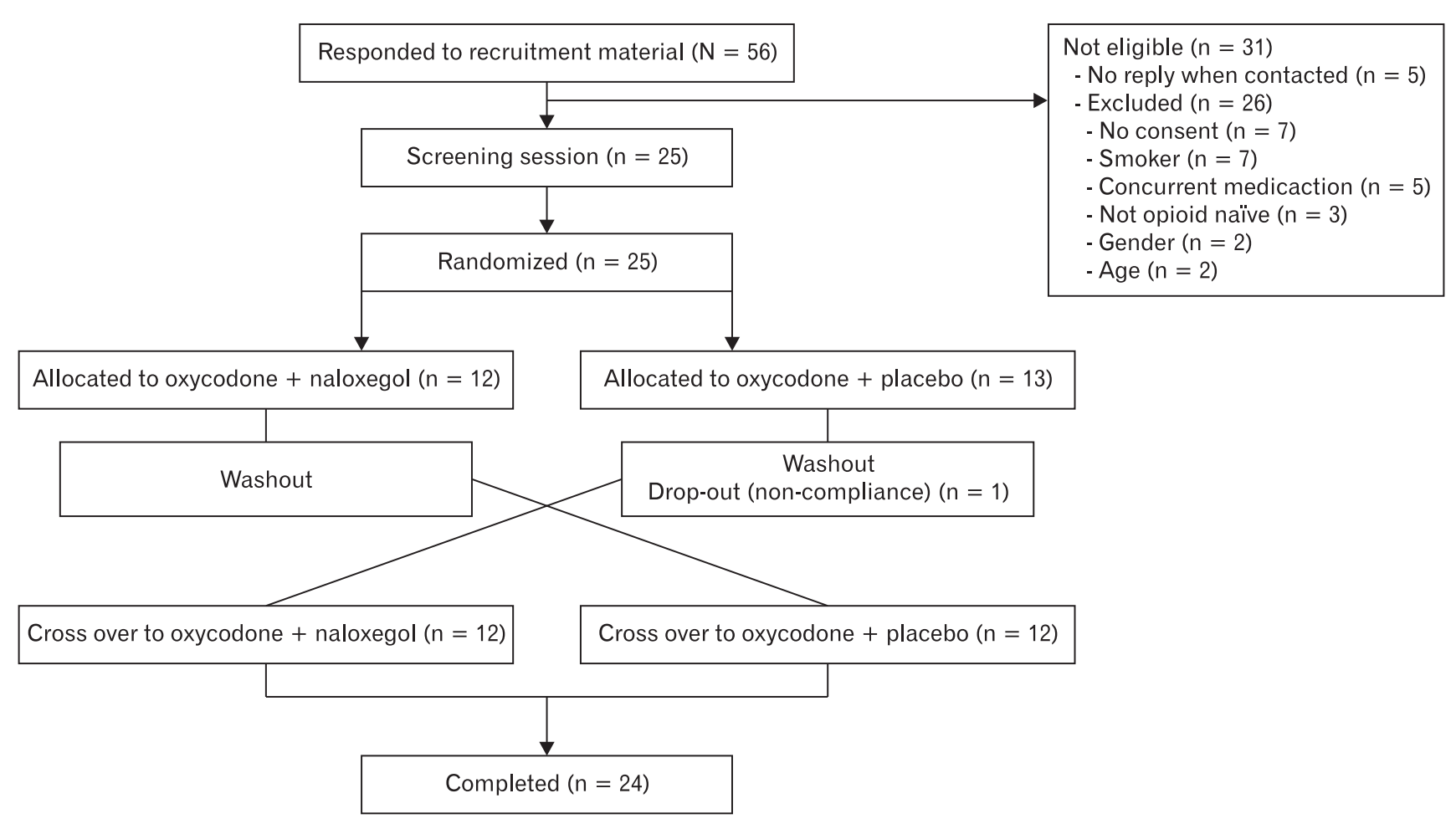

Figure 2. Flowchart for disposition of participants. 
it was not possible to determine segmental colonic transit times in $12 / 48$ recordings ( 7 in the oxycodone + placebo treatment and 5 in oxycodone + naloxegol), due to either poor data quality and/or inadequate data due to capsule battery loss. This left 36 recordings for further analysis of segmental colonic transit. Capsule retention on day 6 occurred in 3/24 recordings during oxycodone + naloxegol treatment and 8/24 during oxycodone + placebo $(P=0.084)$. Naloxegol reduced oxycodone-induced prolongation of total GI transit time by $21 \%$ (56.8 \pm 21.3 hours vs $71.3 \pm 19.8$ hours; $P=$ 0.016 , and colonic transit time by $23 \%$ (45.0 \pm 21.1 hours vs 59.7 \pm 19.3 hours; $P=0.006$ ), compared to placebo. Post-hoc analysis revealed the reduction in colonic transit time by naloxegol to be confined to the rectosigmoid segment (60\%; $9.2 \pm 8.6$ hours vs 23.0 \pm 13.6 hours; $P=0.047)$. There was no difference between oxy- codone + naloxegol and oxycodone + placebo treatments in either gastric emptying ( $5.3 \pm 4.8$ hours vs $3.4 \pm 3$ hours; $P=0.144$ ) or small bowel transit time $(7.4 \pm 2.6$ hours vs $7.9 \pm 5.4$ hours; $P=$ $0.648)$.

\section{Colonic Fecal Volume}

Eleven out of 96 MRI scans were not performed post-treatment due to capsule retention, 4 scans were missed due to technical problems with the MRI scanner, and 2 scans were discarded due to insufficient data quality. Thus, 80 scans were left for further analysis, in which 23 pre-treatment scans were obtained for each treatment, and 15/19 post-treatment scans were obtained for oxycodone + naloxegol and oxycodone + placebo treatment, respectively. Measures of colonic fecal volume are presented in Table 2.

Table 1. Total and Segmental Gastrointestinal Transit Times

\begin{tabular}{lccr}
\hline Variables (hr) & Oxycodone + naloxegol & Oxycodone + placebo & $P$-value \\
\hline Gastric emptying & $5.3(3.1-7.4)$ & $3.4(3.4-4.5)$ & 0.432 \\
Small bowel transit time & $7.4(6.3-8.5)$ & $7.9(5.4-9.6)$ & 0.999 \\
Colonic transit time & $45.0(35.5-53.0)$ & $59.7(52.0-68.3)$ & 0.018 \\
Ascending colon & $16.4(8.8-23.9)$ & $12.2(4.9-19.0)$ & 0.452 \\
Transverse colon & $10.1(5.3-15.0)$ & $10.6(6.8-14.4)$ & 0.231 \\
Descending colon & $4.6(1.7-7.4)$ & $9.0(5.0-13.0)$ & 0.104 \\
Rectosigmoid colon & $9.2(5.0-13.4)$ & $23.0(16.0-30.0)$ & 0.047 \\
Total gastrointestinal transit time & $56.8(48.0-65.7)$ & $71.3(63.0-79.5)$ & 0.016 \\
\hline
\end{tabular}

Data are presented as means (95\% confidence intervals). $n=48$ recordings (24 in each treatment) to determine gastric emptying, small bowel transit time, colonic transit time, and total gastrointestinal transit time. $n=36$ recordings (19 in the naloxegol treatment and 17 in placebo) to determine segmental colonic transit times.

Table 2. Total and Segmental Colonic Fecal Volumes (in $\mathrm{mL}$ )

\begin{tabular}{clcc}
\hline \multicolumn{1}{c}{ Segment } & Assessment & Oxycodone + naloxegol & Oxycodone + placebo \\
\hline Ascending colon & Pre-treatment & $227(196-258)$ & $230(199-261)$ \\
& Post-treatment & $281(236-326)$ & $301(257-345)$ \\
& Difference & $54(3-105)$ & $71(22-120)$ \\
Transverse colon & Pre-treatment & $172(132-211)$ & $188(152-224)$ \\
& Post-treatment & $232(184-281)$ & $244(194-295)$ \\
Descending colon & Difference & $60(2-118)$ & $56(-2-116)$ \\
& Pre-treatment & $95(68-122)$ & $93(64-122)$ \\
Rectosigmoid colon & Post-treatment & $154(116-192)$ & $134(96-172)$ \\
& Difference & $76(33-119)$ & $41(2-84)$ \\
Total colon & Pre-treatment & $139(114-163)$ & $203(147-199)$ \\
& Post-treatment & $149(114-185)$ & $35(-22-93)$ \\
& Difference & $10(-30-40)$ & $680(590-770)$ \\
& Pre-treatment & $634(547-721)$ & $884(739-1030)$
\end{tabular}

${ }^{a}$ Pre-treatment vs post-treatment.

Data are presented as means (95\% confidence intervals). $\mathrm{n}=23$ pre-treatment scans for each treatment period. $\mathrm{n}=19$ and 15 post-treatment scans for oxycodone + naloxegol and oxycodone + placebo treatment period, respectively. 
Fecal volume increased post-treatment, compared to pre-treatment, in both treatments $(29 \%, 818 \pm 273 \mathrm{~mL}$ vs $634 \pm 200 \mathrm{~mL}$ for oxycodone + naloxegol, $P<0.001 ; 30 \%, 884 \pm 262 \mathrm{~mL}$ vs 680 $\pm 207 \mathrm{~mL}$ for oxycodone + placebo, $P<0.001)$. No significant difference in volume post-treatment was found between treatments $(884 \pm 262 \mathrm{~mL}$ vs $818 \pm 273 \mathrm{~mL}, P=0.201$.

\section{Associations to Gastrointestinal Symptoms}

There were no associations between subjectively reported GI symptoms and segmental transit times or colonic fecal volumes (all Pearson's $r< \pm 0.5$; all $P>0.05$ ).

\section{Discussion}

This study investigated the effect of naloxegol on GI transit times and colonic fecal volume during experimentally-induced OIBD. Naloxegol reduced total GI and colonic transit times during oxycodone treatment, whereas gastric emptying and small bowel transit time were unaffected. Naloxegol did not affect fecal volume in the colon.

As the study was part of a larger protocol that also evaluated the effect of naloxegol on anal sphincter function and GI symptoms presented previously, ${ }^{16}$ an overview of results are presented in Table 3.

It was previously demonstrated that oxycodone prolonged total and segmental GI transit times in a trial employing a similar study

Table 3. Overview of Naloxegol Effects

\begin{tabular}{|c|c|}
\hline Outcome & Effect \\
\hline \multicolumn{2}{|l|}{ Effects on anal sphincter function } \\
\hline Anal resting pressure ${ }^{a}$ & $\leftrightarrow$ \\
\hline Sphincter relaxation $^{\mathrm{a}}$ & $\uparrow$ \\
\hline Distensibility of anal canal $^{\mathrm{a}}$ & $\leftrightarrow$ \\
\hline \multicolumn{2}{|l|}{ Effects on subjective scores } \\
\hline PAC-SYM scores $^{a}$ & $\downarrow$ \\
\hline SBM frequency $^{\mathrm{a}}$ & $\leftrightarrow$ \\
\hline SBM stool consistency ${ }^{\mathrm{a}}$ & $\uparrow$ \\
\hline \multicolumn{2}{|c|}{ Effects on GI transit and colonic fecal volumes } \\
\hline Total GI transit time & $\downarrow$ \\
\hline Colonic transit time & $\downarrow$ \\
\hline Gastric emptying & $\leftrightarrow$ \\
\hline Small bowel transit time & $\leftrightarrow$ \\
\hline Fecal volume & $\leftrightarrow$ \\
\hline
\end{tabular}

${ }^{a}$ Results from Grønlund et al. ${ }^{16}$

PAC-SYM, Patient Assessment of Constipation Symptom; SBM, spontaneous bowel movement; GI, gastrointestinal; $\leftrightarrow$, unaffected; $\uparrow$, increased; $\downarrow$, decreased by naloxegol. design. ${ }^{15}$ This indicates that the chosen dose of oxycodone (ie, 30 mg per day) and the length of treatment was sufficient to establish a model of OIBD. The present results support a previous study where naloxegol reduced morphine-induced prolongation of total GI transit time, as assessed with the lactulose hydrogen breath test. $^{22}$ Additionally, our findings are in line with a previous study investigating another commercialized peripherally acting $\mu$-opioid receptor antagonists, alvimopan (only approved in the United States for post-operative ileus), in which a dosage of $12 \mathrm{mg}$ bid reversed codeine-induced prolongation of small bowel and colonic transit. ${ }^{23}$ In contrast, methylnaltrexone (a peripherally acting $\mu$-opioid receptor antagonist only available as subcutaneous injection in advanced OIBD) had no effect on codeine-induced prolongation of colonic transit. ${ }^{24}$ For the latter, the authors question whether a higher dosage of methylnaltrexone may be needed to produce a detectable effect on transit in opioid-naïve participants. It is important to recognize that, compared to eg, radioscintigraphy, the 3D-Transit system essentially tracks a single solid marker throughout the GI tract, and not necessarily the stool itself. Furthermore, a wide variation in GI transit times measured with the 3D-Transit has been found, with a median day-to-day variation in total GI transit time of 10 hours (range of 3-25 hours), between capsules ingested at the same time of the morning the following day. ${ }^{18}$ Thus, the difference in total and segmental GI transit time between oxycodone + naloxegol and oxycodone + placebo treatment needs to be interpreted with caution. However, the 3D-Transit system was the best method of choice as it holds several advantages compared to other available tools. ${ }^{25}$ In addition, transit times obtained from the 3D-Transit system has been validated against radiopaque markers, a well-established and commonly used method. ${ }^{26}$ The normal increase in propagating motor patterns, including high-velocity propagating sequences (also recognized as mass movements) occurring after a meal, are also diminished in patients with slow transit constipation. ${ }^{27}$ These pathological mechanisms may explain why naloxegol only reduced transit time in colon, and not in the stomach and the small bowel.

There were no associations between subjective assessments of constipation and transit times or fecal volumes, and results from another part of the same study also showed that naloxegol did not increase spontaneous bowel movement frequency despite an increase in sphincter relaxation (Table 3 ). ${ }^{16}$ On the other hand, constipation is a subjective phenomenon composed of a series of complaints. Although it is often defined by bowel movement - especially in epidemiological studies - it is primarily characterized by straining, gas, hard consistency of stools, and abdominal discomfort, with infrequent bowel movements and bloating both ranking No. 5 on the 
list. ${ }^{28}$ Similar findings have been found in patients with constipation where spontaneous bowel movement frequency correlated poorly with transit time. ${ }^{29}$ In summary, no significant associations between subjective and objective measurements were found. However, the objective measurements are still relevant as the study was designed to investigate the mechanisms of the effect of naloxegol on experimentally induced OIBD, and the objective methods provide us with more detailed information on intestinal motility, than the subjective assessments.

The fact that naloxegol mainly reduced transit time in the rectosigmoid part of the colon may relate to alleviation of defecation problems collectively known as opioid-induced anal sphincter dysfunction. $^{30}$ This dysfunction may be an indirect consequence of opioid-induced prolonged transit causing increased passive absorption of fluids, ultimately leading to stools difficult to pass. Also, elicitation thresholds for the recto-anal inhibitory reflex have been found to be decreased during opioid treatment, and other results from our study demonstrated that this effect was reverted by naloxegol (Table 3). ${ }^{31,16}$ However, these findings may also have to do with exaggerated continence of promoting motility which should be further investigated in new studies. Normalization of motility and reduced passive absorption of gut fluids may also explain why improvement in self-reported stool consistency by naloxegol was found (Table 3). ${ }^{16}$

The increase in colonic fecal volume after 6 days of oxycodone treatment in both periods verified what has previously been shown. ${ }^{2}$ However, a refined method was used to address non-gaseous fecal volume only, excluding the volume of the colon wall itself and gas within the intestine. Considering that naloxegol reduced colonic transit time, it is interesting that no effect on fecal volume was found. This may relate to the effect of the assessed drugs on gut secretion. When opioids act on $\mu$-receptors within the enteric nervous system, the release of the vasoactive intestinal peptide is blocked, ultimately leading to decreased gut secretion. ${ }^{32}$ Hypothetically, naloxegol may counteract these effects, leading to more watery stool, which may be interpreted as a higher fecal load on the MRI images. This would ultimately counterbalance the fecal volume to equal that of placebo treatment, despite faster GI transit time. This theory is supported by, the analysis of other endpoints from the same study from our group, ${ }^{16}$ and a recent study in opioid-treated patients ${ }^{33}$ where increased scores of stool consistency (ie, softer stools) were found after treatment with naloxegol. However, at the moment, the applied MRI-based method does not allow quantification of water load in colonic content. Additional refinement of the MRI method is needed to provide important knowledge into gut secretion mechanisms, and how this is affected by medication. Missed MRI scans is a major study limitation, in particular in the 8 cases in which the capsule in the placebo arm was retained. In itself, capsule retention indicates constipation, and it is likely that these participants would also show the most pronounced increase in fecal volume. Hence, the data on colonic fecal volume may be systematically underestimated, and the results should therefore be interpreted with caution. Also, it would have been commendable to perform MRI scans on each day, however this was not feasible. As the post-treatment MRI scan was conducted after assessment of transit time, information from days in-between 1 and 6 is unavailable.

Confounding factors like other GI diseases, concomitant drug use, and depression are difficult to avoid in clinical studies. In an experimental OIBD model applied in healthy persons, these factors are profitably controlled for, however, the experimental setting may not completely mirror the clinical picture of chronic opioid users. Therefore, statistical significance in the experimental setting may not translate to clinical relevance. However, one could also speculate that the observed effects of naloxegol on transit time and symptoms would be even more pronounced in patients with OIBD.

So far, the clinical benefit of naloxegol to treat OIBD has predominantly been based on the reduction in spontaneous bowel movement frequency. To gain new insights into the underlying effects of naloxegol, the current study evaluated its effects on total and segmental GI transit times, and colonic fecal volume employing the 3D-Transit system and a novel MRI technique in opioid-treated heathy participants. Naloxegol reduced total GI transit time, and colonic transit time with the main effect on rectosigmoid transit. Taken into account that naloxegol treatment also reduced GI symptoms and improved stool consistency (Table 3), ${ }^{16}$ our results support the use of naloxegol in prevention and treatment of OIBD. Also, these findings add knowledge to the current understanding of peripherally acting $\mu$-opioid receptor antagonist effects on the opioidaffected GI tract.

Acknowledgements: The authors thank radiographer Kenneth Krogh Jensen for valuable help in analyzing MRI scans and study nurse Isabelle M Larsen for practical help in study conduction.

Financial support: This study was funded in part by AstraZeneca Nordic Baltic, Sweden, and in part by the Svend Andersen Foundation, Denmark.

Conflicts of interest: Asbjørn M Drewes received unrestricted grants from AstraZeneca, Shire, Almirall, Grünenthal, and Pfizer. 
Author contributions: Debbie Grønlund contributed to study design, data acquisition, data analysis, data interpretation, and drafting of the manuscript; Anne E Olesen and Asbjørn M Drewes contributed to study supervision, study design, data interpretation, and revising the manuscript; Esben B Mark contributed to data analysis, data interpretation, and revising the manuscript; and Klaus Krogh and Jens B Frøkjær contributed to study design, data interpretation, and revising the manuscript. All authors approved the final version of the manuscript.

\section{References}

1. Wood JD, Galligan JJ. Function of opioids in the enteric nervous system. Neurogastroenterol Motil 2004;16(suppl 2):17-28.

2. Nilsson M, Poulsen JL, Brock C, et al. Opioid-induced bowel dysfunction in healthy volunteers assessed with questionnaires and MRI. Eur J Gastroenterol Hepatol 2016:514-524.

3. Christensen HN, Olsson U, From J, Breivik H. Opioid-induced constipation, use of laxatives, and health-related quality of life. Scand J Pain 2016;11:104-110.

4. Cook SF, Lanza L, Zhou X, et al. Gastrointestinal side effects in chronic opioid users: results from a population-based survey. Aliment Pharmacol Ther 2008;27:1224-1232.

5. Hjalte F, Berggren AC, Bergendahl H, Hjortsberg C. The direct and indirect costs of opioid-induced constipation. J Pain Symptom Manage 2010;40:696-703.

6. Brock C, Olesen SS, Olesen AE, Frøkjaer JB, Andresen T, Drewes AM. Opioid-induced bowel dysfunction: pathophysiology and management. Drugs 2012;72:1847-1865.

7. Drewes AM, Munkholm P, Simrén M, et al. Definition, diagnosis and treatment strategies for opioid-induced bowel dysfunction-recommendations of the nordic working group. Scand J Pain 2016;11:111-122.

8. Holzer P. Non-analgesic effects of opioids: management of opioid-onduced constipation by peripheral opioid receptor antagonists: prevention or withdrawal? Curr Pharm Des 2012;18:6010-6020.

9. Floettmann E, Bui K, Sostek M, Payza K, Eldon M. Pharmacologic profile of naloxegol, a peripherally acting $\mu$-opioid receptor antagonist, for the treatment of opioid-induced constipation. J Pharmacol Exp Ther 2017;361:280-291.

10. Bui K, She F, Sostek M. The effects of renal impairment on the pharmacokinetics, safety, and tolerability of naloxegol. J Clin Pharmacol 2014;54:1375-1382.

11. Bui K, She F, Hutchison M, Brunnström $\AA$, Sostek M. Absorption, distribution, metabolism, and excretion of $\left[{ }^{14} \mathrm{C}\right]$-labeled naloxegol in healthy subjects. Int J Clin Pharmacol Ther 2015;53:838-846.

12. Chey WD, Webster L, Sostek M, Lappalainen J, Barker PN, Tack J. Naloxegol for opioid-induced constipation in patients with noncancer pain. N Engl J Med 2014;370:2387-2396.

13. Webster L, Chey WD, Tack J, Lappalainen J, Diva U, Sostek M. Randomised clinical trial: the long-term safety and tolerability of naloxegol in patients with pain and opioid-induced constipation. Aliment Pharmacol Ther 2014;40:771-779.

14. Webster L, Dhar S, Eldon M, Masuoka L, Lappalainen J, Sostek M. A phase 2, double-blind, randomized, placebo-controlled, dose-escalation study to evaluate the efficacy, safety, and tolerability of naloxegol in patients with opioid-induced constipation. Pain 2013;154:1542-1550.

15. Poulsen JL, Nilsson M, Brock C, Sandberg TH, Krogh K, Drewes AM. The impact of opioid treatment on regional gastrointestinal transit. J Neurogastroenterol Motil 2016;22:282-291.

16. Grønlund D, Poulsen JL, Krogh K, et al. The impact of naloxegol on anal sphincter function - using a human experimental model of opioidinduced bowel dysfunction. Eur J Pharm Sci 2018;117:187-192.

17. Gregersen T, Grønbæak H, Worsøe J, Schlageter V, Laurberg S, Krogh K. Effects of sandostatin LAR on gastrointestinal motility in patients with neuroendocrine tumors. Scand J Gastroenterol 2011;46:895-902.

18. Haase AM, Gregersen T, Schlageter V, et al. Pilot study trialling a new ambulatory method for the clinical assessment of regional gastrointestinal transit using multiple electromagnetic capsules. Neurogastroenterol Motil 2014;26:1783-1791.

19. Nilsson M, Sandberg TH, Poulsen JL, et al. Quantification and variability in colonic volume with a novel magnetic resonance imaging method. Neurogastroenterol Motil 2015;27:1755-1763.

20. Sandberg TH, Nilsson M, Poulsen JL, et al. A novel semi-automatic segmentation method for volumetric assessment of the colon based on magnetic resonance imaging. Abdom Imaging 2015;40:2232-2241.

21. Bendezú RA, Barba E, Burri E, et al. Colonic content in health and its relation to functional gut symptoms. Neurogastroenterol Motil 2016;28:849-854.

22. Eldon MA, Kugler AR, Medve RA, Bui K, Butler K, Sostek M. Safety, tolerability, pharmacokinetics, and pharmacodynamic effects of naloxegol at peripheral and central nervous system receptors in healthy male subjects: a single ascending-dose study. Clin Pharmacol Drug Dev 2015;4:434-441.

23. Gonenne J, Camilleri M, Ferber I, et al. Effect of alvimopan and codeine on gastrointestinal transit: a randomized controlled study. Clin Gastroenterol Hepatol 2005;3:784-791.

24. Wong BS, Rao AS, Camilleri M, et al. The effects of methylnaltrexone alone and in combination with acutely administered codeine on gastrointestinal and colonic transit in health. Aliment Pharmacol Ther 2010;32:884-893.

25. Grønlund D, Poulsen JL, Sandberg TH, et al. Established and emerging methods for assessment of small and large intestinal motility. Neurogastroenterol Motil Published Online First: 13 Jan 2017. doi: 10.1111/ nmo.13008.

26. Gregersen T, Haase AM, Schlageter V, Gronbaek H, Krogh K. Regional gastrointestinal transit times in patients with carcinoid diarrhea: assessment with the novel 3D-transit system. J Neurogastroenterol Motil 2015;21:423-432.

27. Dinning PG, Wiklendt L, Maslen L, et al. Colonic motor abnormalities in slow transit constipation defined by high resolution, fibre-optic manometry. Neurogastroenterol Motil 2015;27:379-388.

28. Johanson JF, Kralstein J. Chronic constipation: a survey of the patient 
perspective. Aliment Pharmacol Ther 2007;25:599-608.

29. Saad RJ, Rao SS, Koch KL, et al. Do stool form and frequency correlate with whole-gut and colonic transit? results from a multicenter study in constipated individuals and healthy controls. Am J Gastroenterol 2010;105:403-411.

30. Tuteja AK, Biskupiak J, Stoddard GJ, Lipman AG. Opioid-induced bowel disorders and narcotic bowel syndrome in patients with chronic non-cancer pain. Neurogastroenterol Motil 2010;22:424-430, e96.
31. Musial F, Enck P, Kalveram KT, Erckenbrecht JF. The effect of loperamide on anorectal function in normal healthy men. J Clin Gastroenterol 1992;15:321-324.

32. Wood JD. Enteric nervous system: reflexes, pattern generators and motility. Curr Opin Gastroenterol 2008;24:149-158.

33. Webster L, Lembo A, Datto C, Sostek M. I7. The effect of naloxegol on stool consistency in patients with noncancer pain and opioid-induced constipation. Pain Manag Nurs 2017;18:74. 